\title{
ISOLEMENT DE BACTÉRIOPHAGES SPÉCIFIQUES POUR DES STREPTOCOQUES LACTIQUES (1)
}

\author{
par \\ MHe J. JOSSE
}

Nous nous sommes proposé, sur les conseils de M. le Pr Lemorane d'entreprendre l'étude des caractères que doivent présenter les streptocoques lactiques pour être de bons ferments lactiques.

Il nous a paru intéressant, tant au point de vue bactériologique qu'au point de vue intérêt pratique, de commencer cette étude par l'action des bactériophages sur les ferments lactiques.

Ce problème des bactériophages des ferments lactiques a été étudié par M. Mazé (2) en France, par Whitehead (3), Hunter (4), Anderson et Meanwell en Angleterre, par Mostmann et Ritter en Suisse.

Les souches de ferments lactiques employées semblent appartenir au groupe Streptococcus lactis bien que certains ne présentent pas tous les caractères propres à ce groupe.

Six des souches $\left(1_{1}, 1_{2}, 1_{4}, 1_{5}, 1_{9}, 1_{10}\right)$ ont été isolées d'une crème de la région d'Isigny, deux autres $\left(B_{3}, B_{2}\right)$ proviennent de beurres de fermes de Normandie, et la dernière $\left(\mathrm{C}_{5}\right)$ d'un beurre fermier de la région de la Somme.

Pour trouver des bactériophages actifs sur ces souches, nous avons entrepris dans le laboratoire de M. le $\mathrm{D}^{\mathrm{r}} \mathrm{WAHL}$, et sur ses conseils, les recherches suivantes:

1. Recherche du pouvoir lysogène des souches de ferments lactiques. Le fait a été observé une seule fois pour une des souches par rapport à une autre $\left(B_{2}\right.$ sur $\left.l_{9}\right)$ et n'a pu être reproduit.

2. Isolement des phages à partir de l'eau d'égout. Les premiers isolements de bactériophages furent faits à partir de l'eau d'égout (Clichy) ; la méthode d'isolement est la suivante : les neuf souches sont groupées trois par trois, chaque groupe de trois est ensemencé dans un ballon contenant $30 \mathrm{~cm}^{3}$ d'eau peptonée glucosée à $30 \%$ et $30 \mathrm{~cm}^{3}$ d'eau d'égout. On laisse les trois ballons cinq heures à l'étuve à $35^{\circ}$.

On prélève quelques centimètres cubes de chaque ballon dans un tube, les trois tubes sont portés au bain-marie à $56^{\circ}$ pendant une demi-heure pour tuer les germes. On a ainsi trois préparations pou-

(1) Société française de Microbiologie, 3 avril 1947; in Annales Institut Pasteur, LXXIII, 1947, 906.

(2) P. Mazé. Comptes rendus de la Société de Biologie, 1941, 135, 807.

(3) H, R. Whitehead et G. J. E. Hunter. Journal Dairy Res., 1939, 10, 403, 409,

(4) G. J. E. Hunter. Journal Hyg., 1946, 44, no 4. 
vant contenir des phages. Une goutte de chacun est étalée sur plaque de gélose nutritive glucosée avec chacune des souches de ferments lactiques correspondantes et mises à l'étuve. On observe un phénomène de lyse totale dans huit boîtes, une seule boîte ensemencée avec la souche $1_{2}$ n'est pas lysée. On en conclut que l'eau d'égout contient des bactériophages spécifiques des ferments lactiques utilisés.

On obtient des plages distinctes en employant des dilutions de trois préparations sur les ferments lactiques. Chaque boîte présente un mélange de plages de taille différente. L'isolement des phages a été fait par la méthode des isolements en série à partir d'une plage. Six isolements au minimum ont été faits pour chaque bactériophage. Ils ont été considérés comme purs après ces six isolements et après avoir obtenu une seule plage sur chaque boîte ou du moins quelques plages de même taille très éloignées les unes des autres. Malgré cela, dans plusieurs cas, la taille des plages n'était pas uniforme et il semble que certains phages donnent des plages de taille inégale.

Un bactériophage à très grandes plages n'a pu être isolé par cette méthode mais a demandé une technique spéciale sur laquelle nous reviendrons dans une publication ultérieure.

Ces grandes plages qui interféraient et couvraient la superficie de la boîte masquaient d'autres bactériophages à plages de taille différente ; il en a été en effet isolé deux autres sur la même préparation. D'autre part, sur ces très grandes plages apparaissaient des cultures secondaires qui étaient lysées par un bactériophage de plus petite taille: On a obtenu la lyse des souches sensibles par leurs bactériophages en eau peptonée ou en bouillon, un seul de petite taille agissant sur la souche $1_{5}$ ne s'est pas multiplié quel que soit le milieu employé ; eau peptonée ou bouillon, les deux milieux étant glucosés ou non.

Au total 11 souches de bactériophages ont été isolées.

Il fallait voir si ces 11 souches appartenaient à différentes races. Pour l'établir nous avons étudié :

10 La gamme d'action sur les neuf souches de ferments lactiques;

20 La résistance croisée.

1. Le tableau suivant donne la gamme d'action ; on voit que les souches de phages peuvent se diviser en cinq groupes, chaque groupe étant actif sur les mêmes souches de ferments lactiques.

Seule la souche de ferments lactiques $1_{2}$ est résistante à ces phages. Les souches $1_{1}, I_{5}, C_{5}$ se montrent sensibles à un plus grand nombre de phages que les souches $1_{4}, 1,1_{1}, B_{3}, B_{2}$.

2. Pour l'étude de la résistance croisée, l'isolement des souches 
GAMMES D'ACTION DES SOUGHES DES BAGTÉRIOPHAGES SUR LES SOUGHES DE FERMENTS LACTIQUES

\begin{tabular}{|c|c|c|c|c|c|c|c|c|c|c|c|}
\hline \multirow{2}{*}{$\begin{array}{l}\text { Apparition } \\
\text { des cultures } \\
\text { secondaires }\end{array}$} & \multirow{2}{*}{$\begin{array}{c}\text { Taille } \\
\text { des plages }\end{array}$} & \multirow{2}{*}{$\begin{array}{l}\text { Souches } \\
\text { de bacté- } \\
\text { riophages }\end{array}$} & \multicolumn{9}{|c|}{ Souches de Ferments lactiques } \\
\hline & & & $\left(1_{1}\right)$ & $\left(1_{2}\right)$ & $\left|\left(1_{4}\right)\right|$ & $\left(1_{5}\right)$ & $\left|\left(1_{0}\right)\right|$ & $\left|\left(I_{10}\right)\right|$ & $\left(\mathrm{B}_{3}\right)$ & $\left(\mathrm{B}_{2}\right)$ & $\left(\mathrm{C}_{5}\right)$ \\
\hline $4^{\mathrm{e}}$ jour ..... & petite & $1 \mathrm{~L}$ & + & & + & + & & & & 4 & + \\
\hline $4^{e}$ jour ...... & moyenne & $1 \mathrm{~L}^{\prime}$ & + & & 1 & + & & & & & + \\
\hline 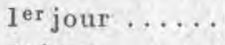 & moyenne & $5 \mathrm{~L}$ & & (6) & & & + & 1 & + & & \\
\hline $4^{\mathrm{e}}$ jour ...... & moyenne & $6 \mathrm{~L}$ & & & & & + & + & + & & \\
\hline $4^{\mathrm{e}}$ jour $\ldots \ldots$ & petite & $7 \mathrm{~L}^{\prime}$ & 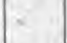 & & & & 4 & + & + & & \\
\hline $4^{\mathrm{e}}$ jour. . . . & moyenne & $7 \mathrm{~L}^{\prime}$ & & & & & + & + & + & & \\
\hline $4^{e}$ jour...... & pl. auréol. & $8 \mathrm{La}$ & + & & + & + & & & & + & + \\
\hline $4^{\mathrm{e}}$ joux $\ldots \ldots$ & grande & $8 \mathrm{Lg}$ & + & & + & + & & & & + & + \\
\hline $4^{e}$ jour $\ldots \ldots$. & moyenne & $8 \mathrm{~L}^{\prime}$ & + & & + & + & & & & + & + \\
\hline $3^{\mathrm{e}}$ jour ....... & petite & $8 \mathrm{~L}$ & + & & + & + & & & & + & + \\
\hline $3^{e}$ jour ...... & petite & $9 \mathrm{~L}$ & + & & & f & & & & & + \\
\hline
\end{tabular}

résistantes à chaque souche de bactériophage a été fait, puis chaque souche résistante a été mise en contact avec les bactériophages présentant la même gamme d'action sur les mêmes couches de ferments lactiques, aucun phénomène de lyse n'a été observé.

D'après l'étude de la gamme d'action et de la résistance croisée on peut conclure presque certainement que les souches de bactériophages agissant sur les mêmes souches de ferments lactiques appartiennent à la même race. Au total nous aurions cinq races de bactériophages. Ce travail est une première étape et ces bactériophages vont nous servir pour étudier leur action sur les ferments lactiques.

(Institut Pasteur, Service du Bactériophage et Service des Fermentations.)

\section{BIBLIOGRAPHIE ANALYTIQUE}

\section{LES LIVRES}

Rapport pour les trois années 1944, 1945 et 1946. Institut National de Recherches de laiterie. Université de Reading. Stimulation de la production laitière chez les vaches par l'alimentation avec protéine iodurée. 1 brochure de 94 pages.

Comme suite aux indications portées dans le précédent Rapport pour 1941-1943, on donne les résultats suivants obtenus en 1944-1946: 10 la perte. de poids produite par l'alimentation avec caséine iodurée chez les vaches en période lactée peut être évitée en donnant des aliments supplémentaires. 